\title{
Editorial Statistics 2019
}

Papers submitted

Of those papers were:

- rejected by editorial decision 98

- handed back to authors for revision (by editorial decision) 14

- submitted to double-blind review after initial revision 2

- rejected by editorial decision after initial revision 2

- rejected by unanimous reviewer decision --

- withdrawn by the authors 1

- cancelled by editorial decision 3

- altered to Research Notes 1

- directly submitted to double-blind review 60

- altered to Research Notes 1

- rejected by unanimous reviewer decision 8

- rejected by editorial decision 3

- withdrawn by the authors 5

- cancelled by editorial decision --

- Rejection rate: $\quad 66 \%$

- accepted for publication (after revision) 12

- published as Articles in 2019

- published as Research Notes in 2019

- scheduled to be published in $2020 \quad 4$

Average feedback duration

(i.e. time between submission of a paper and feedback):

Feedback loops of more than 100 days 55

(Editorial committee target line):

Reviews provided: 205 
We express our gratitude to the following reviewers for their support provided to our journal through their critical and fair reviews in 2019:

Ulun Akturan

Maria Aluchna

Maike Andresen

Aylin Ataay

Norbert Bach

Gyula Bakacsi

David Balkin

Yannick Bammens

Pieter-Jan Bezemer

Daniel Blab

Yury Blagov

Susanne Blazejewski

Suleika Bort

Dorota Bourne

Carina Braun

Markus Braun

Zoltan Buzady

Doina Catana

Tomaž Čater

Sven-Olof Collin

Barbara Covarrubias Venegas

Bruno Dallago

Claudia Doblinger

Diana Eerma

Jacob Eisenberg

Allen Engle

John Erpenbeck

Bengi Ertuna

Andreas Falke

Tom Falter

Stuart Farquhar

Marion Festing

Simon Fietze

Peter Fischer

Marzena Fryczyńska

Hansjörg Gaus

Anja Geigenmüller

Eskil Goldeng

Jan Goldenstein
Olaf Kranz

Irena Kustec

Marina Latukha

Kari Liuhto

Shyqyri Llaci

Albert Löhr

Susan Madsen

Matjaz Maletic

Milan Maly

Wenzel Matiaske

Wolfgang Mayrhofer

Regina Mulder

Zlatko Nedelko

Ivan Novy

Nóra Obermayer

Krzysztof Obloj

Simon Oertel

Andrei Panibratov

Daniel Pastuh

Aron Perenyi

Günther Pernul

Claudia Petrescu

Gregor Pfajfar

Alessia Pisoni

Evgeny Polyakov

Julia Ponomareva

József Poór

Theo Postma

Danijel Pucko

Judith Pütter

Philipp Ramin

Olaf Rank

Matevz Raskovic

Kerstin Rego

Anna Remisova

Natalia Ribberink

Alessandra Rigolini

Elisabetta Rocchiccioli

Katja Rost 
Victoria Golikova

Igor Gurkov

Axel Haller

Sebastian Händschke

Stefan Hauptmann

Balázs Heidrich

Roland Helm

Niels Hermes

Graham Hollinshead

Christina Hoon

Annette Hoxtell

Michael Hunoldt

Stefan Hüsig

Akihiro Ishikawa

Steffen Jahn

Andreja Jaklic

Barbara Jankowska

Jatta Jännari

Miroslaw Jarosinski

Stephan Kaiser

Päivi Karhunen

Antti Kauppinen

Ralf Kellner

Shannon Kerwin

Wafa Khlif

Fabian Kindermann

Mirjana Kljajic

Heiko Kohlenbecker

Romana Korez Vide

Mihai Korka

Marko Kosak

Arjan Kozica
Hartmut Rumpf

Jelle Schepers

Stefan Schmid

Arnold Schuh

Klaus-Peter Schulz

Alma Sehic

Anna Soulsby

Tobias Specker

Zivile Stankeviciute

Stefanie Steinhauser

Simona Sternad

Johannes Steyrer

Thomas Straub

Erna Szabo

Till Talaulicar

Loreta Tauginiene

Susanne Tietze

Piotr Trąpczyński

Jeannette Trenkmann

Maaja Vadi

Marco van Gelderen

Emil Velinov

Johan Vögel

Marta Vohlidalova

Dieter Wagner

Marius Wehner

Aleksandra Wasowska

Elke Weik

Michael Whitall

Stefan Wüst

Anju Yu

Marek Zielinski

Editorial Committee

Thomas Steger

Irma Rybnikova

Rainhart Lang 